\title{
A Case of Phyllodes Tumor with Focal Transition into Low-Grade Lymphangiosarcoma
}

\author{
Thomas Bachleitner-Hofmann ${ }^{a}$ Sebastian F. Schoppmann ${ }^{a} \quad$ Margaretha Rudas $^{b}$ \\ Peter Birner $^{b}$ Helene Wiener ${ }^{b}$ Peter Dubsky ${ }^{a}$ Peter Blaha ${ }^{a}$ Emanuel Sporn ${ }^{a}$ Peter Panhofer ${ }^{a}$ \\ Florian Fitzal $^{\mathrm{a}}$ Sebastian Roka ${ }^{\mathrm{a}}$ Daniela Kandioler ${ }^{\mathrm{a}}$ Michael Gnant ${ }^{\mathrm{a}}$ Raimund Jakesz ${ }^{\mathrm{a}}$
}

a Department of Surgery,

${ }^{b}$ Department of Pathology, Medical University of Vienna, Austria

\author{
Key Words \\ Phyllodes tumor - Malignant transformation . \\ Lymphangiosarcoma
}

\section{Summary}

Background: Phyllodes tumors are fibroepithelial neoplasms that are extremely rare, comprising less than $1 \%$ of all breast neoplasms. Malignant transformation may occur, usually within the stromal component of the phyllodes tumor. The most common patterns of stromal malignancy are fibrosarcoma and myxoliposarcoma. Other patterns include chondrosarcoma, osteosarcoma and leiomyosarcoma. Case Report: Here, we report the case of a 42-year-old female patient who had a borderline phyllodes tumor with focal transition into a low-grade lymphangiosarcoma. The patient was treated with mastectomy and sentinel lymph node biopsy which showed no metastatic spread to the axilla. No further adjuvant treatment was given, and the patient remains free of recurrent disease 9 months after surgery. Conclusions: To the best of our knowledge, this is the first report of a malignant transformation of a borderline phyllodes tumor into a lymphangiosarcoma.

\section{Introduction}

Phyllodes tumors are fibroepithelial neoplasms that have the potential to recur $[1,2]$. They are extremely rare, comprising less than $1 \%$ of all breast neoplasms. Classification into benign, borderline and malignant phyllodes tumors depends on

\author{
Schlüsselwörter \\ Phyllodestumor · Maligne Transformation . \\ Lymphangiosarkom
}

\section{Zusammenfassung}

Hintergrund: Phyllodestumore sind extrem seltene fibroepitheliale Neoplasien, die insgesamt weniger als $1 \%$ der Brusttumore darstellen. Eine maligne Transformation ist möglich und findet üblicherweise innerhalb der Stromakomponente des Phyllodestumors statt. Die häufigsten histologischen Differenzierungstypen sind Fibrosarkom und Myxoliposarkom, seltener Chondrosarkom, Osteosarkom und Leiomyosarkom. Fallbericht: Wir berichten von einer 42 Jahre alten Patientin mit einem Borderline-Phyllodestumor mit fokalem Übergang in ein niedrig malignes Lymphangiosarkom. Die Behandlung erfolgte mittels Mastektomie und Sentinel-Lymphknotenbiopsie, die keinen Hinweis auf Metastasierung in die Axilla ergab. Es wurde keine weitere adjuvante Therapie verabreicht, und die Patientin ist 9 Monate nach der Operation rezidivfrei. Schlussfolgerung: Unseres Wissens handelt es sich hierbei um den ersten Fallbericht einer malignen Transformation eines Phyllodestumors in ein Lymphangiosarkom. several histologic criteria, such as stromal hypercellularity, pleomorphism and mitotic activity, as well as pushing versus infiltrative margins [3]. Malignant transformation usually occurs within the stromal component of the phyllodes tumor, although there have been rare cases where the epithelium showed signs of carcinoma [1,4]. The two most common

\begin{tabular}{ll}
\hline KARGER & ( 2006 S. Karger GmbH, Freiburg \\
Fax +49 7614520714 & Accessible online at: \\
$\begin{array}{l}\text { E-mail Information@Karger.de } \\
\text { www.karger.com }\end{array}$ & www.karger.com/brc \\
&
\end{tabular}


Fig. 1. Mammography and ultrasound imaging: a mammography at initial presentation showed a well-demarcated lesion $2.5 \mathrm{~cm}$ in diameter without microcalcifications in the upper inner quadrant of the right breast; $\mathbf{b}$ ultrasound at initial presentation showed a 2.5 -cm hypoechoic lesion with well-defined borders and posterior enhancement; $\mathbf{c}$ ultrasound 2 months after initial presentation showed an increase in tumor size to $5.0 \mathrm{~cm}$.
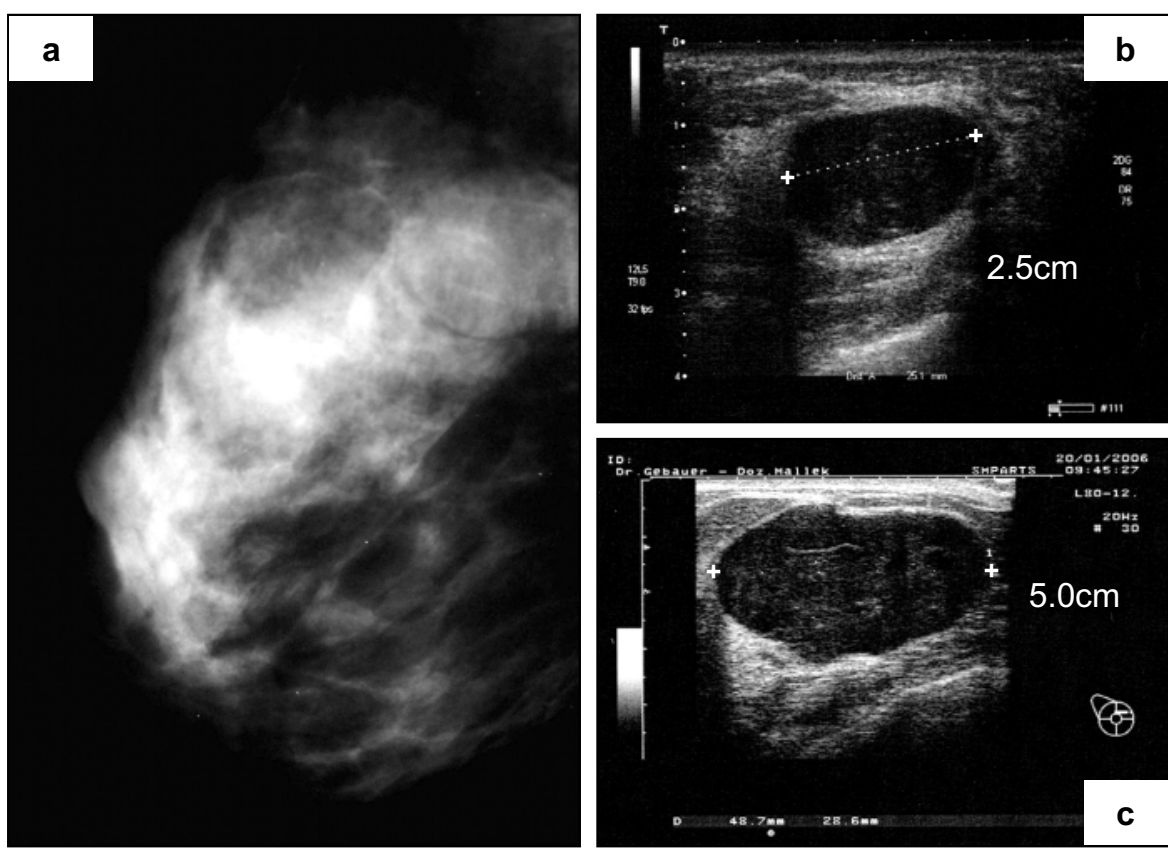

patterns of malignant transformation of the stroma are fibrosarcoma and myxoliposarcoma. Other patterns of stromal malignancy include chondrosarcoma, osteosarcoma and leiomyosarcoma. Here, we report the case of a 42 -year-old female patient who had a bordeline phyllodes tumor with focal transition into a low-grade lymphangiosarcoma.

\section{Case Report}

We report the case of a 42-year-old female patient who presented with a palpable mass in the upper inner quadrant of the right breast. Physical examination revealed a firm, discrete and mobile mass, approximately $2.5 \mathrm{~cm}$ in diameter. The clinical axillary lymph node status was negative. The family history revealed 1 case of invasive breast cancer in a seconddegree relative. Mammography showed a well-demarcated $2.5-\mathrm{cm}$ lesion without microcalcifications, and breast ultrasound showed a hypoechoic lesion with well-defined borders and posterior enhancement (figs. 1a, b). A diagnosis of fibroadenoma was made, and the lesion was categorized as BIRADS-III. 2 months after initial presentation, the tumor had grown to a diameter of $5.0 \mathrm{~cm}$ (fig. 1c). Core needle biopsy revealed a fibroepithelial neoplasm with the characteristic features of a phyllodes tumor. A quadrantectomy of the upper inner quadrant was performed, and an additional $1-\mathrm{cm}$ lesion in the lower inner quadrant which had been detected intraoperatively was also removed. Because of an unfavorable tumor-tobreast ratio, a modified Hall-Findlay mammoplasty was performed to improve cosmetic outcome. The immediate postoperative course was uneventful, and the patient was discharged from hospital on the third postoperative day.

Histologic examination of the specimen showed a typical phyllodes tumor with cystic ducts and leaf-like papillary protrusions. The stroma displayed moderate cellularity, moderate pleomorphism and myxoid degeneration with stromal edema. No infiltration of the adjacent breast parenchyma was noted. Resection margins were free. Histologic examination of the additional lesion in the lower inner quadrant gave similar results. Notably, the larger tumor in the upper inner quadrant (but not the tumor in the lower inner quadrant) additionally displayed irregular slit-like vascular spaces scattered over large areas of the tumor compatible with pseudoangiomatous stromal hyperplasia. While the majority of vascular spaces was lined by CD34-positive, spindle-like cells without atypia, several vascular conglomerates were lined by pleomorphic cells with nuclear atypia and increased mitotic activity. In one area of the tumor, vascular spaces were absent and the atypic cells formed a solid tumor cell nest with $>6$ mitoses per high-power field (HPF) (fig. 2). Immunohistochemistry of this area revealed that the tumor cells stained positive for the specific lymphatic endothelium marker podoplanin but negative for CD34 and the myoepithelial markers calponin and muscle-specific actin [HHF-35]. Staining for the proliferation marker Ki-67 revealed $20 \%$ positive cells. Based on these findings, a diagnosis of a borderline phyllodes tumor with focal transition into a low-grade lymphangiosarcoma was made.

In view of the lymphangiosarcoma, the unfavorable tumor-to-breast ratio and an extensive delayed postoperative hematoma which had developed by the 10th postoperative day, mastectomy with sentinel lymph node biopsy was performed 2 weeks after the initial operation. Histologic examination of the mastectomy specimen showed no further phyllodes tumor and sentinel node biopsy revealed no evidence for metastatic spread to the axilla. Interestingly, the remaining breast parenchyma displayed extensive stromal fibrosis with a vast number of ectatic capillaries and surrounding cavernous lymph vessels, lined by endothelial cells without atypia or mitotic activity. The vascular spaces were found across the entire remaining breast parenchyma, indicative of a non-neoplastic, hamartomatous angiomatosis. The vascular changes were also found around peripheral and large milk ducts which were filled with blood, fibrin and hemosiderin-laden macrophages, indicative of recurrent intraluminal bleeding (figs. 3a, b).

The postoperative course after mastectomy was uneventful. Staging using computed tomography and bone scan showed no evidence of metastatic spread. The patient received no further adjuvant treatment. 9 months after surgery, the patient remains free of recurrent disease.

\section{Discussion}

To the best of our knowledge, this is the first report of a lymphangiosarcoma arising within the stroma of a phyllodes tu- 

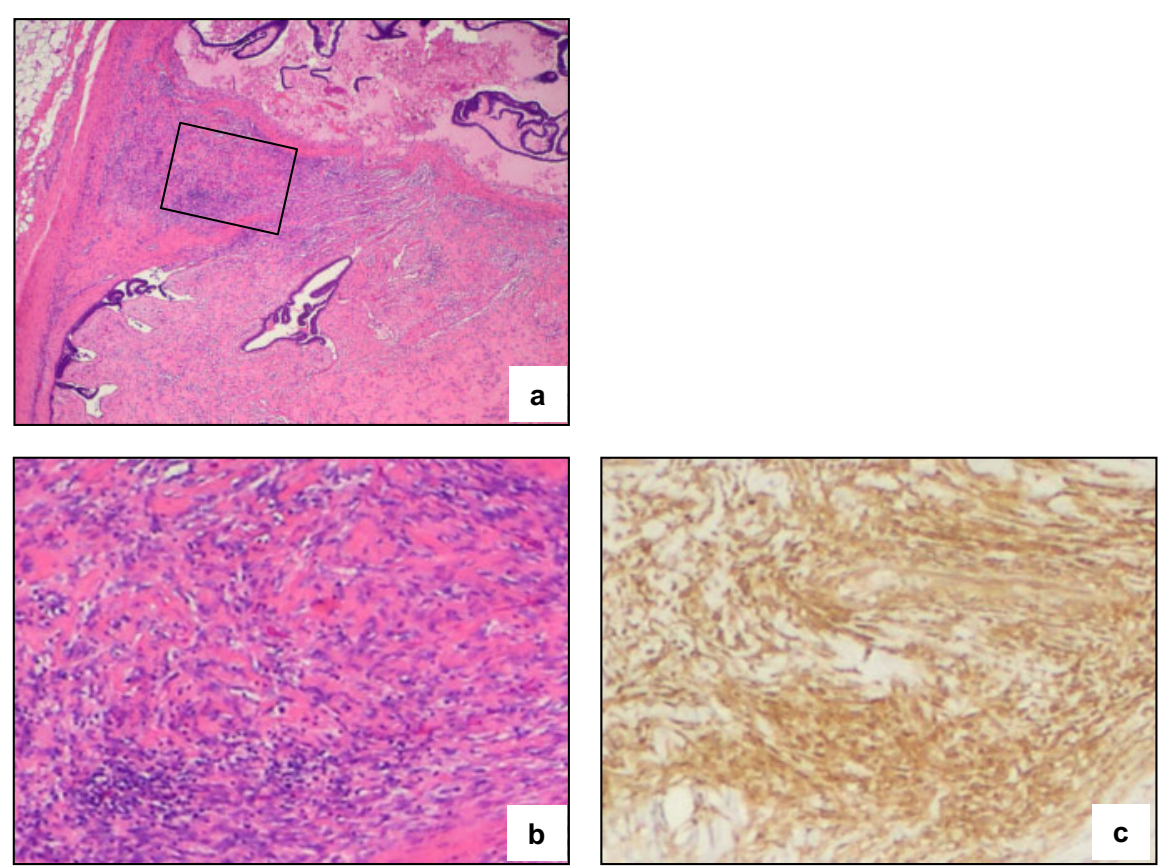

Fig. 2. Immunohistochemical staining of podoplanin, CD34 and Ki-67 proteins: a $\mathrm{H}$ and $\mathrm{E}$ section $(40 \times)$ of the phyllodes tumor with focal lymphangiosarcoma; the rectangular region delineates the area shown on the other panels; b $\mathrm{H}$ and $\mathrm{E}$ section; c serial section stained by anti-podoplanin antibody showing podoplaninpositive tumor cells; $\mathbf{d}$ serial section stained by anti-CD34 antibody showing negativity of the tumor cells for CD34; e serial section stained by anti-Ki67 antibody showing approximately $20 \%$ positive cells.
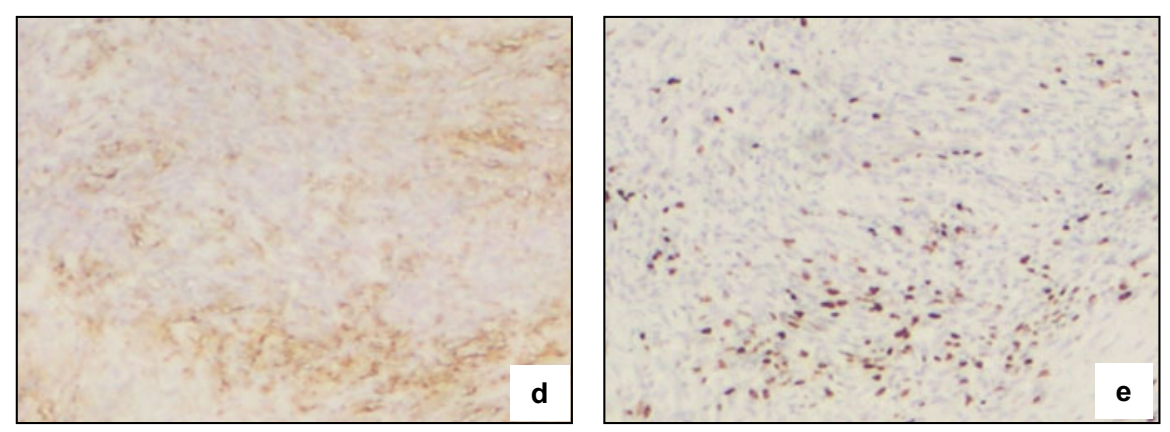

mor. It is well known that phyllodes tumors may display in creased cellularity with pleomorphism, nuclear atypia and malignant transformation of the stroma, rendering them borderline or malignant phyllodes tumors [1]. The most common patterns of malignant transformation are fibrosarcoma and myxoliposarcoma. Other differentiation patterns include chondrosarcoma, osteosarcoma and leiomyosarcoma. Frequently, areas of malignant transformation are focal and may be overlooked if careful sampling is not performed $[5,6]$. In the present case, the malignant stromal cells showed differentiation into a low-grade lymphangiosarcoma, staining positive for the highly specific lymphovascular endothelial marker podoplanin while staining negative for CD34 and the myoepithelial markers calponin and muscle-specific actin [HHF-35]. Notably, the phyllodes tumor displayed pseudoangiomatous stromal hyperplasia, a proliferation of the mammary stroma with formation of a complex pattern of slit-like pseudovascular spaces, which has previously been described in phyllodes tumors [7, 8]. Although we cannot provide definite evidence, it seems likely that the lymphangiosarcoma originated from the pseudoangiomatous stromal hyperplasia within the phyllodes tumor. Even though pseudoangiomatous stromal hyper- plasia has not been related to malignancy so far, there has been a report of lobular angiomatosis of the breast progressing to angiosarcoma approximately 1 year after diagnosis [9]. Generally, the treatment of choice for phyllodes tumors is wide local excision without axillary staging [1]. However, for histologically malignant phyllodes tumors, some authors have recommended mastectomy as routine initial treatment [10-12]. In the present case, treatment initially consisted of wide local excision with a modified Hall-Findlay mammoplasty owing to an unfavorable tumor-to-breast ratio. However, when permanent section analysis revealed a lymphangiosarcoma within the phyllodes tumor, we eventually decided to perform a mastectomy with sentinel node biopsy. Reasons for the more radical treatment approach were as follows: First, there are no currently available data on the local recurrence rate and metastatic potential of a lymphangiosarcoma arising within a phyllodes tumor, suggesting that mastectomy may be advantageous compared to wide local excision for reasons of oncologic safety. Second, despite an uneventful immediate postoperative course, the patient developed a massive delayed hematoma by the 10th postoperative day, which required extensive surgical revision. Importantly, the postoperative course after mastecto- 
Fig. 3. $\mathrm{H}$ and $\mathrm{E}$ staining of normal breast tissue after mastectomy: a $\mathrm{H}$ and $\mathrm{E}$ staining $(40 \times)$ showing the hamartomatous angomatosis with ectatic capillaries within a fibrous stroma; b $\mathrm{H}$ and $\mathrm{E}$ staining $(40 \times)$ showing ectatic capillaries surrounded by cavernous lymph vessels; note the large milk duct filled with blood, indicative of recurrent intraluminal bleeding.
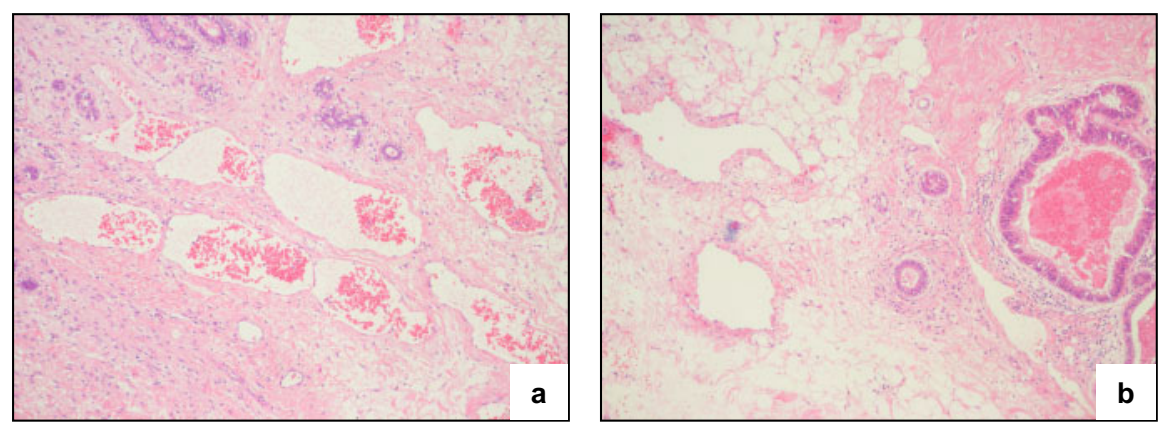

my was uneventful, and histology showed no evidence of residual disease in the breast or metastatic spread to the axilla.

It is unclear which factors led to the massive delayed hematoma in our patient. Although a surgery-related technical problem cannot be ruled out with certainty, we feel that this is unlikely in view of the fact that the immediate postoperative course of our patient was uneventful and that the patient was discharged from hospital on the 3 rd postoperative day in good clinical condition. Furthermore, thrombocyte levels as well as plasmatic coagulation tests were normal, ruling out an underlying coagulopathy. Notably, however, the remaining breast parenchyma displayed a hamartomatous angiomatosis with a vast number of ectatic capillaries and surrounding cavernous lymph vessels. The vascular abnormalities were also found around milk ducts which were filled with blood and hemosiderin-laden macrophages, indicative of recurrent intraluminal bleeding. Although we cannot provide definitive evidence, it seems likely in our opinion that the hamartomatous angiomatosis could have led to an inherent bleeding tendency and could thus have contributed to the delayed postoperative hematoma in our patient.

An important aspect of our report is the fact that it under- scores the diagnostic value of core needle biopsy in presumably benign lesions of the female breast. On mammography and ultrasonography, the appearance of phyllodes tumors is usually similar to that of large fibroadenomas, which often leads to delayed diagnosis of the phyllodes tumor. While fine needle aspiration has been reported to be inadequate for early diagnosis of phyllodes tumors because of a false-negative rate of up to $86 \%[13,14]$, core needle biopsy correctly identified the lesion in our patient as a phyllodes tumor. Therefore, we feel that core needle biopsy should be given preference over fine needle aspiration for the diagnostic workup of large, presumably benign lesions of the breast.

In conclusion, we have reported for the first time the emergence of a lymphangiosarcoma from the stromal component of a high-grade phyllodes tumor. The patient was treated with radical mastectomy and sentinel node biopsy. No adjuvant treatment was given, and the patient remains free of recurrence 9 months after surgery. Of particular importance is that our report underscores the diagnostic accuracy of core needle biopsy which should be given preference over fine needle aspiration for the differential diagnosis of presumably benign lesions of the female breast.

\section{References}

1 Petrek JA: Phyllodes tumors, in Harris JR, Lippmann ME, Morrow M, Osborne CK (eds): Diseases of the Breast. Philadelphia, PA, Lippincott Williams and Wilkins, 2000, pp. 669-75.

$\checkmark 2$ Feakins RM, Mulcahy HE, Nickols CD, Wells CA: p53 expression in phyllodes tumours is associated with histological features of malignancy but does not predict outcome. Histopathology 1999;35:162-9.

$\checkmark 3$ Moffat CJ, Pinder SE, Dixon AR, Elston CW, Blamey RW, Ellis IO: Phyllodes tumours of the breast: a clinicopathological review of thirty-two cases. Histopathology 1995;27:205-18.

4 Rosen PP: Coexistent mammary carcinoma and cystosarcoma phyllodes. Breast 1975;1:9-15.

5 Treves N, Sunderland DA: Cystosarcoma phyllodes of the breast: a malignant and a benign tumor; a clinicopathological study of seventy-seven cases. Cancer 1951;4:1286-1332.
6 Lester J, Stout AP: Cystosarcoma phyllodes. Cancer 1954;7:335-53.

7 Vuitch MF, Rosen PP, Erlandson RA: Pseudoangiomatous hyperplasia of mammary stroma. Hum Pathol 1986;17:185-91.

8 Khoury T, Hurd T, Tan D: Phyllodes tumor with pseudoangiomatous stroma hyperplasia. Breast $\mathrm{J}$ 2005;11:285-7.

9 Breitfellner G: On the relation between lobular angiomatosis and angiosarcomas of the breast. Z Krebsforsch Klin Onkol 1975;84:345-50.

10 Norris HJ, Taylor HB: Relationship of histologic features to behavior of cystosarcoma phyllodes. Analysis of ninety-four cases. Cancer 1967;20: 2090-9.
Kessinger A, Foley JF, Lemon HM, Miller DM: Metastatic cystosarcoma phyllodes: a case report and review of the literature. J Surg Oncol 1972;4: 131-47.

12 Christensen L, Nielsen M, Madsen PM: Cystosarcoma phyllodes. A review of 19 cases with emphasis on the occurrence of associated breast carcinoma. Acta Pathol Microbiol Immunol Scand [A] 1986;94:35-41.

13 Salvadori B, Cusumano F, Del Bo R, Delledonne V, Grassi M, Rovini D, Saccozzi R, Andreola S Clemente C: Surgical treatment of phyllodes tumors of the breast. Cancer 1989;63:2532-6.

14 Stanley MW, Tani EM, Rutquist LE, Skoog L: Cystosarcoma phyllodes of the breast: a cytologic and clinicopathologic study of 23 cases. Diagn Cytopathol 1989;5:29-34. 